\title{
Construção e validação de recursos audiovisuais para motivar pessoas com hipertensão ao uso de anti-hipertensivos
}

\section{Construction and validation of audiovisual resources to motivate people with hypertension to use} antihypertensives

\section{Construcción y validación de recursos audiovisuales para motivar a las personas con hipertensión para utilizar antihipertensivos}

Taciana da Costa Farias Almeida ${ }^{1}$ (1)

Mailson Marques de Sousa² Bernadete de Lourdes André Gouveia ${ }^{1}$ (D) Simone Helena dos Santos Oliveira ${ }^{3}$ (1)

1. Universidade Federal de Campina Grande, Unidade Acadêmica de Enfermagem. Campina Grande, PB, Brasil.

2. Hospital Municipal Santa Isabel. João Pessoa, PB, Brasil.

3. Universidade Federal da Paraíba, Programa de Pós-Graduação em Enfermagem. João Pessoa, PB, Brasil.

\begin{abstract}
REsUmo
Objetivo: Construir e validar comunicação audiovisual e mensagens persuasivas para motivar o uso de anti-hipertensivos. Método: Estudo metodológico baseado nas crenças de pessoas com hipertensão arterial sistêmica em relação ao tratamento medicamentoso, tendo o suporte da Teoria do Comportamento Planejado. Adotaram-se os princípios de Fleming e da Persuasão para desenvolvimento da comunicação audiovisual e mensagens. 13 juizes especialistas realizaram a validação de conteúdo, analisada pelo Índice de Validade de Conteúdo. Resultados: A comunicação audiovisual apresenta 3 minutos e 58 segundos de duração e 71 telas. Foram consideradas válidas 29 mensagens persuasivas ilustradas, sendo 15 positivas e 14 negativas Conclusão e implicações para a prática: A comunicação audiovisual e as mensagens persuasivas foram consideradas válidas, adequadas e com capacidade de persuasão para motivar o uso de anti-hipertensivos orais. Pesquisa de intervenção faz-se necessária para testar o efeito dos recursos audiovisuais com intenção de realizar o comportamento.
\end{abstract}

Palavras-chave: Hipertensão; Anti-Hipertensivos; Comunicação Persuasiva; Recursos Audiovisuais.

\section{Abstract}

Objective: Building and validating audiovisual communication and persuasive messages to motivate the use of antihypertensive drugs. Method: Methodological study based on the beliefs of people with systemic arterial hypertension in relation to drug treatment, supported by the Theory of Planned Behavior. The principles of Fleming and Persuasion were adopted for the development of audiovisual communication and messages. 13 expert judges performed the content validation, analyzed by the Content Validity Index. Results: the audiovisual communication has 3 minutes and 58 seconds in duration and 71 screens. Twenty-nine persuasive illustrated messages were considered valid, 15 positive and 14 negative messages. Conclusion and implications for practice: Audiovisual communication and persuasive messages were considered valid, adequate and capable of persuasion to motivate the use of oral antihypertensives. Intervention research is necessary to test the effect of audiovisual resources with the intention of carrying out the behavior.

Keywords: Hypertension; Antihypertensive drugs; Persuasive communication; Audiovisual resources.

\section{Resumen}

Objetivo: Construir y validar la comunicación audiovisual y los mensajes persuasivos para motivar el uso de antihipertensivos. Método: Estudio metodológico basado en las creencias de las personas con hipertensión arterial sistémica en relación con el tratamiento farmacológico, apoyado por la Teoría del Comportamiento Planificado. Los principios de Fleming y la Persuasión fueron adoptados para el desarrollo de la comunicación y los mensajes audiovisuales. 13 jueces expertos realizaron la validación del contenido, analizada por el Índice de Validez del Contenido. Resultados: La comunicación audiovisual tiene 3 minutos y 58 segundos de duración y 71 pantallas. Se consideraron válidos 29 mensajes ilustrativos persuasivos, 15 mensajes eran positivos y 14 negativos. Conclusión e implicaciones para la práctica: La comunicación audiovisual y los mensajes persuasivos se consideraron válidos, adecuados y capaces de persuadir para motivar el uso de antihipertensivos orales. La investigación de intervención es necesaria para probar el efecto de los recursos audiovisuales con la intención de llevar a cabo el comportamiento. Palabras clave: Hipertensión; Drogas anti-hipertensivas; Comunicación persuasiva; Recursos audiovisuales.
Autor correspondente:

Taciana da Costa Farias Almeida.

E-mail: tacianacfalmeida@gmail.com.

Recebido em 16/04/2020.

Aprovado em 07/08/2020.

DOl:https://doi.org/10.1590/2177-9465-EAN-2020-0127 


\section{INTRODUÇÃO}

A adesão ao tratamento medicamentoso da hipertensão arterial sistêmica (HAS) é considerada complexa e de difícil manejo pela pessoa com hipertensão. Tal fato ocorre por ser um tratamento de longo prazo, com presença de efeitos adversos, custo considerável e, por vezes, desagradável, o que incorre na falta de adesão. ${ }^{1}$ Assim, torna-se importante desenvolver estratégias motivadoras, baseadas em teoria, para fortalecer e incentivar a tomada dos anti-hipertensivos. ${ }^{2}$

A Teoria do Comportamento Planejado (TCP) pressupõe que o comportamento humano é predito pela intenção (motivação) e influenciado por três determinantes psicossociais e respectivas crenças: atitude, formada por crenças comportamentais, resultados prováveis do comportamento e avaliações desses resultados pelos indivíduos; norma subjetiva, constituída por crenças normativas sobre a opinião de pessoas significativas e a motivação para atender a essas expectativas; e controle comportamental percebido, inerente às crenças de controle sobre a presença de fatores que possam facilitar ou impedir o desempenho do comportamento. Portanto, intervenções destinadas a mudar/fortalecê-lo podem ser dirigidas a um ou mais dos seus determinantes e, uma vez que mudanças ocorram nesses fatores, devem produzir transformações nas intenções comportamentais. $^{3}$

Ao considerar que a medida da intenção é influenciada por crenças relevantes sobre o comportamento, uma intervenção é bem-sucedida quando produz alteração nessa medida; e, para mudá-la, é necessário atingir as crenças que guiam o fenômeno de interesse. Entre as intervenções sugeridas pela TCP para modificar crenças e intenção, destaca-se a persuasão. ${ }^{3-5}$

Persuasão é o poder de influenciar e motivar pessoas a aderir a um determinado propósito, que pode utilizar-se de comunicação e mensagens para alterar atitudes, crenças ou comportamentos. ${ }^{6}$ Assim, o alvo da comunicação persuasiva é oferecer informações relevantes a um público específico, a fim de mudar algumas de suas crenças para modular a intenção comportamental. ${ }^{5}$

Contudo, não há diretrizes gerais que determinem quais tipos de informações devem ser incluídas nas mensagens para maximizar mudanças desejadas nas crenças primárias sobre o comportamento. As informações podem apresentar forma breve ou longa, impressa, em áudio, audiovisual ou interativa, e ser usadas para atingir um público amplo e a um custo relativamente baixo. ${ }^{5}$ Podem-se incluir mensagens com diferentes enquadramentos (positivas ou negativas) a fim de influenciar crenças, atitudes, normas subjetivas e controle percebido e, consequentemente, a concepção de uma intenção favorável à realização do comportamento. ${ }^{4}$

A aplicação de mensagens e/ou comunicações persuasivas a comportamentos relacionados à saúde têm evidenciado incentivo e manutenção de comportamentos preventivos, ${ }^{7-9}$ influência no deslocamento de crenças $^{10,11}$ e variação na intenção comportamental. ${ }^{12,13}$ No cenário das doenças cardiovasculares, foram encontrados estudos que desenvolveram mensagens persuasivas para promoção de atividade física, ${ }^{14}$ e obtenção de adesão às recomendações terapêuticas e de prevenção entre indivíduos com doença arterial coronariana. ${ }^{10}$

No âmbito da HAS - doença considerada problema de saúde pública que afeta cerca de $22 \%{ }^{15}$ de pessoas adultas no mundo e $32,5 \%$ no Brasil, ${ }^{16}$ identifica-se um estudo que avaliou o impacto de mensagens positivas (vantagens e benefícios) e negativas (desvantagens ou danos) na decisão de usar antihipertensivos para diminuir o risco de doença cardiovascular nos próximos 10 anos. Todavia, as informações tinham o objetivo de apenas alertar sobre as consequências do uso ou não dos medicamentos prescritos. ${ }^{17}$

No Brasil, não foram identificados estudos sobre o desenvolvimento de comunicação e mensagens persuasivas dirigidas para o comportamento "uso de anti-hipertensivos orais", apesar das evidências de baixa adesão de pessoas com HAS ao tratamento, o que implica desfechos desfavoráveis na manutenção da saúde e da qualidade de vida. ${ }^{1}$

Considerando a problemática da HAS para a saúde pública e a necessidade de incorporar tecnologias leves como estratégias adjuvantes para potencializar a adesão terapêutica e, consequentemente, reduzir complicações e a prevalência da doença, recursos audiovisuais com conteúdo persuasivo estruturados com base nas crenças, preferências e entendimento da terapêutica instituída dessa população devem ser o foco das ações de saúde que ultrapassem estratégias tradicionais de intervenção.

Nessa perspectiva, torna-se relevante construir tecnologias atrativas que motivem a manutenção de comportamentos saudáveis e reforcem aspectos importantes relacionados ao processo saúde/doença e bem-estar, com base nas crenças sobre o uso de anti-hipertensivos orais.

Logo, acredita-se que tecnologias inovadoras como as apresentadas neste estudo poderão dar suporte ao enfermeiro nas estratégias educativas e de cuidado para maximizar a adesão à terapêutica de pessoas com HAS, de modo a alcançar a estabilidade dos níveis pressóricos alterados. Diante do exposto, objetivou-se construir e validar a comunicação audiovisual e mensagens persuasivas para motivar o uso de anti-hipertensivos.

\section{MÉTODO}

Estudo de desenvolvimento metodológico, realizado no período de dezembro de 2018 a julho de 2019, em duas etapas distintas e interrelacionadas, conforme descrito a seguir:

1. a etapa: Os pressupostos da $\mathrm{TCP}^{3-5}$ recomendam que, para a estruturação de mensagens persuasivas, os argumentos sejam fundamentados nas crenças primárias que sustentam o comportamento de interesse pelo grupo destinado a recebêla. Nesse sentido, realizou-se estudo prévio ${ }^{18}$ com o objetivo de analisar as crenças comportamentais, normativas e de controle, relacionadas ao tratamento medicamentoso para HAS. Participaram 28 pessoas com HAS em seguimento em um ambulatório de cardiologia.

As crenças obtidas foram categorizadas por meio da análise de frequências e conteúdo, elegendo-se as modais salientes 
(emitidas com frequência igual a superior a cinco) para compor a comunicação e as mensagens persuasivas, que incluíram: Crenças comportamentais (controlar a pressão, evitar complicações e morte, sentir-me bem, evitar sintomas da doença, segurança e tranquilidade em relação à minha saúde, efeitos adversos/ desagradáveis, ser um tratamento para o resto da vida); Crenças normativas (filhos, esposo(a), médica(o), família); e Crenças de controle (adquirir os comprimidos gratuitamente, o ato de tomar os comprimidos, baixo custo dos medicamentos prescritos, esquecimento do horário, quando tem que comprar). ${ }^{18}$

2. a etapa: Foram elaboradas a comunicação audiovisual e as mensagens persuasivas, e realizada a validação de conteúdo por especialistas. Esta etapa consolidou-se em três momentos distintos, conforme as recomendações de Fleming: ${ }^{19}$ pré-produção, produção e pós-produção.

1. momento - Pré-produção: ocorreu com a construção do roteiro textual da comunicação e das mensagens persuasivas (positivas e negativas) pela pesquisadora principal e elaboração do Storyboard, ou seja, o plano de orientação para a produção dos elementos audiovisuais. ${ }^{20}$ Foram consideradas as crenças eliciadas em estudo prévio, ${ }^{18}$ os seis princípios da persuasão (reciprocidade, consistência, autoridade, validade social, escassez e atração) e as características dos elementos que devem constituí-las (frases concisas, claras e atrativas, imagens, ideia do texto vocabulário sugestivo e adequado ao público-alvo). ${ }^{6}$

O Storyboard foi estruturado no Microsoft Word ${ }^{\circledR}$, dentro de um quadro com duas colunas, constando na primeira coluna trechos da mensagem persuasiva elaborada e a ser ilustrada e na segunda coluna os recursos audiovisuais sugeridos para serem utilizados (cores, imagens, animações, textos, narração e sons de fundo).

2. momento - Produção: consistiu na implementação das ideias elaboradas na pré-produção (Storyboard). ${ }^{19,20}$ Esta fase foi realizada pela pesquisadora, em conjunto com uma profissional da arte e mídia, com experiência em desenvolvimento de tecnologias para comunicação. As cenas da comunicação audiovisual foram criadas com base nos trechos da comunicação textual e ilustradas por imagens próprias do banco de vetores da profissional desenvolvedora, utilizando-se os softwares Adobe Photoshop ${ }^{\circledR}$, Adobe Illustrator ${ }^{\circledR}$ e Sony Vegas $\mathrm{PRO}^{\circledR}$. Para esse desenvolvimento, foi observada a legislação referente aos direitos autorais de utilização e de reprodução de recursos audiovisuais. ${ }^{21}$

Nesta fase, foram escolhidas as cores, fundos de telas, mensagens, animações, música de fundo, sons e imagens ilustrativas do vídeo e mensagens. Foram aplicadas cores mescladas entre tons pastel e cores mais fortes, animações, troca de tela, trilha e efeitos sonoros e mensagem final, estimulando a capacidade de executar o comportamento. A narração inserida no vídeo foi realizada, de forma voluntária, por um profissional da comunicação.

A comunicação audiovisual apresenta informações e argumentos persuasivos e motivacionais sobre aspectos relacionados à HAS como conceito da doença, aspectos fisiológicos, fatores de risco e efeitos colaterais de fármacos. As crenças foram incorporadas, ressaltando a importância da adesão ao tratamento, contemplando reforços positivos das vantagens de tomar os anti-hipertensivos e atenuando possíveis desvantagens. Destacou-se a relevância dos referentes sociais positivos como agentes motivadores para a realização do comportamento, ressaltaram-se as facilidades e minimizaram-se as dificuldades de adesão ao tratamento medicamentoso. O vídeo apresentou duração de 4 minutos e 15 segundos e 84 cenas.

Para as mensagens, também se utilizaram argumentos relacionados às crenças emitidas, os quais foram desenvolvidos em formato de tela de aplicativo. Optou-se por um conceito clean, adotando-se o fundo de tela azul para as positivas, por transmitir positividade, harmonia e serenidade, com o intuito de estimular o lado positivo de tomar os comprimidos prescritos; e o vermelho foi escolhido para o fundo das mensagens negativas, uma vez que esta cor sugere alerta, intensificação e fortalecimento de informação, que, no caso, mostram as consequências de não tomar os comprimidos.

A escolha da cor da fonte amarela nas mensagens teve o propósito de chamar a atenção para as palavras por parte dos espectadores, realçando-se em caixa alta as palavras de destaque nas crenças, além de esta cor ter caráter estimulante, o que se ajusta ao objetivo das mensagens elaboradas.

As mensagens persuasivas foram estruturadas com base nas recomendações do modelo teórico $0^{5} \mathrm{em} 32$ telas em formatos de aplicativo, sendo 16 positivas e 16 negativas, constituídas de textos curtos com imagens e emojis alusivos ao conteúdo das crenças emitidas e com, no máximo, 20 palavras. As mensagens positivas buscam reforçar nas crenças, as vantagens e avaliação das consequências de adotar o comportamento desejado, a motivação do indivíduo em concordar com referentes sociais positivos e sensibilizar para as facilidades do uso de antihipertensivos. As negativas destacam as desvantagens em não adotar o comportamento de usar os anti-hipertensivos orais.

Cabe mencionar que, as mensagens negativas foram elaboradas de modo espelhado, ou seja, o conteúdo textual era similar à comunicação positiva. A título de exemplo, se o conteúdo da mensagem positiva fosse: "Tomando os comprimidos para a hipertensão arterial corretamente, você aumenta as chances de controlar a doença", a redação de maneira negativa seria: "Deixar de tomar os comprimidos para hipertensão arterial dificulta o controle da doença e pode fazer você se sentir mal".

3. ำmento - Pós-Produção: envolveu a edição, com auxílio de profissional de mídias, e a validação de conteúdo do vídeo e mensagens por juízes especialistas nasáreas de conhecimento. ${ }^{19,22,23}$ Estes foram selecionados mediante o atendimento de um dos seguintes critérios: atuar na área Tecnologias da Informação e Comunicação e Arte e Mídia; prestar assistência e desenvolver 
pesquisas dirigidas à pessoa com HAS; e realizar ensino e pesquisa com abrangência da TCP.

Após o levantamento dos nomes dos especialistas, por meio de consulta ao currículo na Plataforma Lattes e leitura de artigos científicos prévios de suas autorias em alguma dessas áreas, foram encaminhados convites via correio eletrônico (e-mail) a 23 especialistas. Destes, 13 concordaram em participar do processo de validação de conteúdo.

$\mathrm{Na}$ sequência, foram encaminhados, via plataforma do Google Docs ${ }^{\circledR}$, o Termo de Consentimento Livre e Esclarecido, os recursos audiovisuais produzidos e o instrumento para análise de conteúdo. Solicitou-se aos participantes, o retorno do material avaliado em um prazo de 30 dias.

No instrumento destinado à análise de conteúdo (vídeo e mensagens), consideraram-se as seguintes propriedades: Abrangência (adequadamente coberto pelo conjunto de itens avaliados - conteúdo, linguagem, público-alvo da comunicação, ilustrações, narração, tempo de duração, potencial persuasivo avaliado); clareza (se os atributos persuasivos estavam descritos de forma compreensível); pertinência (se expressavam verdadeira relação com a proposta do estudo em questão); e persuasão (se podiam ser consideradas persuasivas). ${ }^{22,23}$

Cada propriedade foi avaliada por meio de escala do tipo Likert, com pontuação que variou de 1 a 4, na qual: 1 = Discordo Totalmente (não relevante/não representativo/não claro); 2 = Discordo (necessita de grande revisão para ser representativo/pouco claro); 3 = Concordo (pequena revisão para ser representativo/ bastante claro); 4 = Concordo Totalmente (relevante/representativo/ muito claro). Ao final do instrumento, um espaço foi destinado para a inclusão voluntária de sugestões e/ou modificações, independente da análise realizada, com o intuito de melhorar as comunicações persuasivas. ${ }^{23}$

Utilizou-se o cálculo do Índice de Validade de Conteúdo (IVC), que mede a proporção ou porcentagem de especialistas que estão em concordância com o conteúdo do material avaliado. ${ }^{24,25}$ O IVC foi calculado por meio da soma do número de itens que receberam pontuação "3" ou "4", divididos pelo número total de respostas. Para classificar o item avaliado como válido, considerou-se o IVC $\geq 0,8$. Itens que obtiveram IVC $<0,8$ foram excluídos e/ou reformulados. ${ }^{23}$ Cabe salientar que, mesmo obtendo um IVC válido, todas as construções foram reavaliadas e acatadas as sugestões de modificações, quando consideradas pertinentes pelos pesquisadores.

O estudo integra a pesquisa "Efeito de comunicações persuasivas na intenção comportamental de pacientes hipertensos em tomar os comprimidos prescritos para o controle da hipertensão arterial", aprovada pelo Comitê de Ética em Pesquisa local (Parecer n. ㅇ 2.446.615/2017 e CAAE: 79671317.3.00005782). Todos os participantes autorizaram sua participação mediante a assinatura do Termo de Consentimento Livre e Esclarecido. Em relação aos direitos autorais das imagens utilizadas, obedeceu-se à Lei n. ${ }^{\circ} 9.610$, de 19 de fevereiro de 1998.

\section{RESULTADOS}

Os argumentos utilizados nas comunicações e nas mensagens persuasivas, para influenciar o comportamento "tomar os comprimidos prescritos para controlar a pressão arterial", foram estruturados a partir das crenças modais salientes, oriundas de um estrato representativo do grupo pesquisado, conforme explicitado previamente.

Para o processo de validação das comunicações e mensagens, participaram 13 especialistas que atenderam um ou mais critérios de inclusão delineados, sendo 11 enfermeiros, 1 fisioterapeuta e 1 profissional das mídias visuais. Referente à titulação, foram 11 doutores, um mestre e um pós-doutor. Quanto à atuação, 11 estavam envolvidos em docência e pesquisa, 1 em centro de simulação realística e 1 no desenvolvimento de mídias digitais. Cabe salientar que os especialistas residiam em diferentes estados federativos do Brasil, a saber: Rio Grande do Sul, São Paulo, Minas Gerais, Ceará e Paraíba.

Os aspectos avaliados, as sugestões, assim como os índices de validade de conteúdo atribuídos pelos especialistas, estão descritos na Tabela 1. É possível notar que, mesmo com índice de concordância máximo para itens 1, 2, 5, 6, 7, 9 e 10, os especialistas apresentaram algumas sugestões quanto à aparência, narração e som, duração do vídeo e informações.

As alterações mais sugeridas ocorreram em relação à(ao): escurecimento das cores das telas para contrastar com a cor da letra branca dos escritos na tela, como apresentado na tela abaixo "afasta os sintomas"; adição de legendas nas telas; imagem do medicamento, que na versão inicial, era apresentada como cápsula; imagem do profissional 'médico' para equipe de saúde; e diminuição do tempo de exibição do vídeo. As sugestões foram acatadas e a nova versão do vídeo ficou com 3 minutos e 58 segundos de duração e 71 telas. Algumas telas produzidas são apresentadas na Figura 1.

$\mathrm{Na}$ Tabela 2, são apresentados os Índices de Validade de Conteúdo inerentes à análise, pelos especialistas, das mensagens persuasivas positivas e negativas, nas quais trechos destacam as crenças que subsidiaram a sua construção.

Os resultados revelam que as 16 mensagens positivas e 16 negativas podem contribuir como estratégia de persuasão ao uso dos anti-hipertensivos, ser utilizadas como tecnologia de cuidado pelos profissionais de saúde e pelo indivíduo com hipertensão e, além disso, estão adequadamente cobertas pelo conjunto de itens (conteúdo, linguagem, público-alvo, ilustrações, figuras e potencial persuasivo), obtendo IVC $\geq 0,80$ para os aspectos analisados, com exceção do conteúdo da mensagem 3 , que obteve IVC <0,80 e foi reformulado.

As principais sugestões referiram-se à(s): mudança do termo "hipertensão arterial" para "pressão arterial" na mensagem 3, relacionada à crença "sentir-se bem"; imagens utilizadas, como, por exemplo, adicionar imagem de comprimidos e não de cápsulas; acrescentar pessoas mais jovens, com o objetivo de demonstrar que não só idosos podem apresentar HAS; inserir mensagens curtas, com no máximo 20 palavras, a fim de evitar que mensagens longas dispersem a atenção do usuário; escurecer a cor do fundo da tela, para contrastar com o branco e amarelo da fonte, associado à utilização de emojis, no intuito de intensificar o apelo persuasivo, melhorar a escrita de algumas mensagens, e assim proporcionar adequada compreensão pelo público-alvo. Alguns exemplos são ilustrados na Figura 2. 
Tabela 1. Distribuição dos Índices de Validade de Conteúdo (IVC) atribuídos pelos especialistas aos aspectos avaliados no vídeo e sugestões, de acordo com as crenças emitidas pelos indivíduos com hipertensão arterial. Campina Grande/Paraíba, Brasil, 2019. $(n=13)$

\begin{tabular}{|c|c|c|}
\hline Aspectos analisados & IVC Itens & Sugestões dos Especialistas \\
\hline $\begin{array}{l}\text { 1. A comunicação é apropriada para o } \\
\text { público-alvo? }\end{array}$ & 1,00 & Narração mais pausada e legenda para surdos. \\
\hline 2. A linguagem é de fácil compreensão? & 1,00 & Explicar o que é hipertensão arterial. \\
\hline $\begin{array}{l}\text { 3. As cores e as formas das ilustrações são } \\
\text { adequadas? }\end{array}$ & 0,77 & $\begin{array}{l}\text { Escurecer as telas que apresentam letras brancas, utilizar } \\
\text { cores com mais destaque, alterar a imagem do cérebro. }\end{array}$ \\
\hline $\begin{array}{l}\text { 4. A disposição das figuras está em harmonia } \\
\text { com o texto? }\end{array}$ & 0,77 & $\begin{array}{l}\text { Alterar a imagem da forma farmacêutica cápsula e colocar de } \\
\text { comprimido. }\end{array}$ \\
\hline $\begin{array}{l}\text { 5. As ilustrações são relevantes para a } \\
\text { compreensão do conteúdo? }\end{array}$ & 1,00 & $\begin{array}{l}\text { Foi sugerida a alteração de algumas imagens para tornar a } \\
\text { comunicação mais convincente. }\end{array}$ \\
\hline $\begin{array}{l}\text { 6. A narração está adequada para o } \\
\text { conteúdo? }\end{array}$ & 1,00 & $\begin{array}{l}\text { Melhorar o ruído/abafamento que foi apresentado no vídeo, } \\
\text { assim como o descompasso na pausa da leitura, e manter } \\
\text { o tom de voz, dando maior entonação às informações mais } \\
\text { relevantes. }\end{array}$ \\
\hline $\begin{array}{l}\text { 7. A comunicação é expressa de modo } \\
\text { persuasivo? }\end{array}$ & 1,00 & $\begin{array}{l}\text { Diminuir o tamanho do vídeo, uma vez que, longo, torna-se } \\
\text { cansativo. }\end{array}$ \\
\hline 8. O tempo de duração é satisfatório? & 0,77 & $\begin{array}{l}\text { É longo. Pensar em versões mais curtas que direcionem para o } \\
\text { vídeo completo, como uma estratégia de divulgação. }\end{array}$ \\
\hline $\begin{array}{l}\text { 9. A comunicação contribui como estratégia } \\
\text { de persuasão para a mudança de crenças e } \\
\text { comportamentos protetores? }\end{array}$ & 1,00 & $\begin{array}{l}\text { Acrescentar informações sobre lembrar-se de tomar os } \\
\text { medicamentos. }\end{array}$ \\
\hline $\begin{array}{l}\text { 10. Pode ser utilizada como tecnologia de } \\
\text { cuidado pelos profissionais de saúde e pelo } \\
\text { indivíduo com hipertensão? }\end{array}$ & 1,00 & $\begin{array}{l}\text { Inserir informações que ajudem o indivíduo hipertenso a } \\
\text { tomar os medicamentos. }\end{array}$ \\
\hline
\end{tabular}

Fonte: Dados da Pesquisa, 2019
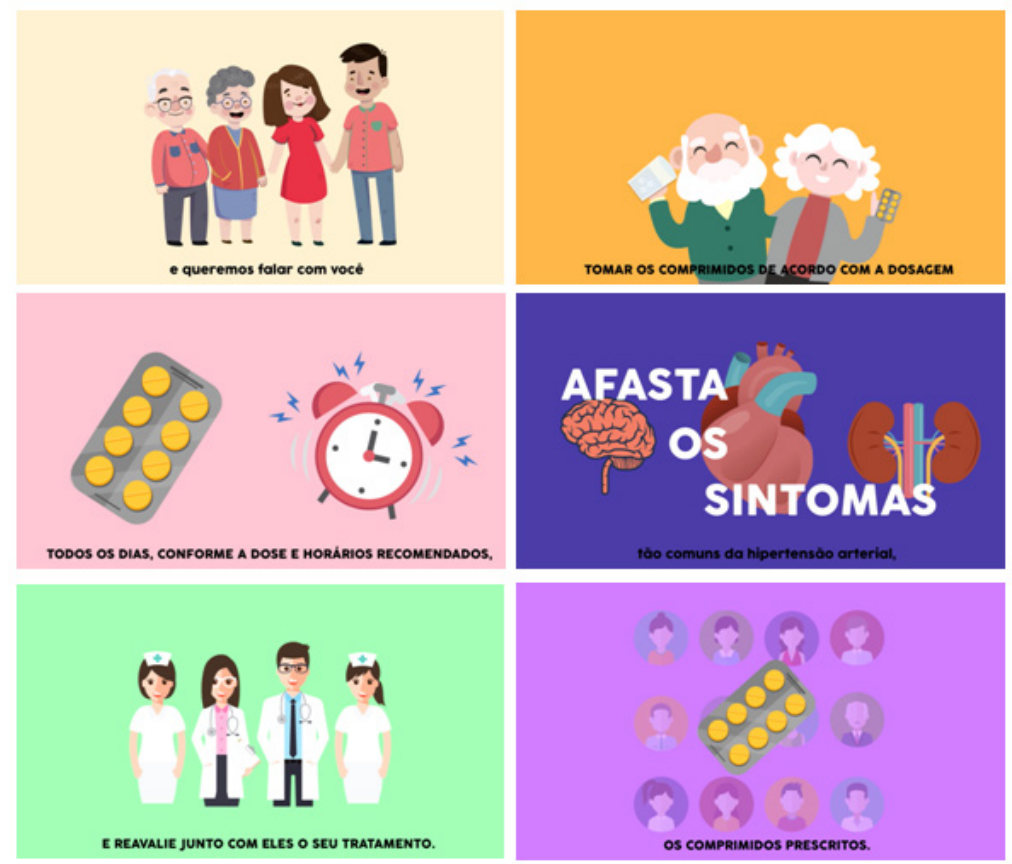

Figura 1. Imagens das telas do vídeo após análise dos especialistas e modificações realizadas. Campina Grande/Paraíba, Brasil, 2019. Fonte: Elaboração própria 
Tabela 2. Distribuição dos Índices de Validade de Conteúdo (IVC) atribuídos pelos especialistas aos aspectos avaliados nas mensagens positivas e negativas, de acordo com as crenças emitidas pelos indivíduos com hipertensão. Campina Grande/ Paraíba, Brasil 2019. ( $\mathrm{n}=13)$

\begin{tabular}{|c|c|c|c|c|c|c|}
\hline \multirow{2}{*}{$\begin{array}{l}\text { Crenças utilizadas na construção das } \\
\text { mensagens persuasivas }\end{array}$} & \multicolumn{3}{|c|}{ Mensagens POSITIVAS } & \multicolumn{3}{|c|}{ Mensagens NEGATIVAS } \\
\hline & $\mathrm{R} / \mathrm{I}$ & Cont. & IVC & $\mathrm{R} / \mathrm{l}$ & Cont. & IVC \\
\hline \multicolumn{7}{|l|}{ Crenças comportamentais } \\
\hline 1. Controlar a pressão arterial & 1,00 & 1,00 & 1,00 & 0,92 & 0,92 & 0,92 \\
\hline 2. Evitar complicações da doença e morte & 1,00 & 1,00 & 1,00 & 1,00 & 1,00 & 1,00 \\
\hline 3. Sentir-se bem & 1,00 & 0,77 & 0,88 & 0,92 & 0,92 & 0,92 \\
\hline 4. Evitar sintomas da doença & 1,00 & 1,00 & 1,00 & 1,00 & 1,00 & 1,00 \\
\hline 5. Sentir-se seguro e tranquilo & 1,00 & 1,00 & 1,00 & 1,00 & 1,00 & 1,00 \\
\hline 6. Efeitos adversos (desagradáveis) & 0,92 & 1,00 & 0,96 & 0,92 & 1,00 & 0,96 \\
\hline 7. Dependente do tratamento & 1,00 & 1,00 & 1,00 & 1,00 & 1,00 & 1,00 \\
\hline \multicolumn{7}{|l|}{ Crenças Normativas } \\
\hline 8. Filhos & 1,00 & 1,00 & 1,00 & 0,92 & 1,00 & 0,96 \\
\hline 9. Esposo(a) & 1,00 & 1,00 & 1,00 & 0,92 & 1,00 & 0,96 \\
\hline 10. Médico(a) & 0,84 & 1,00 & 0,92 & 0,92 & 1,00 & 0,96 \\
\hline 11. Família & 1,00 & 1,00 & 1,00 & 1,00 & 1,00 & 1,00 \\
\hline \multicolumn{7}{|l|}{ Crenças de Controle } \\
\hline 12. Adquirir gratuitamente & 0,92 & 1,00 & 0,96 & 1,00 & 1,00 & 1,00 \\
\hline 13. Tomar por via oral & 0,92 & 1,00 & 0,96 & 0,77 & 0,84 & 0,80 \\
\hline 14. Baixo custo & 1,00 & 1,00 & 1,00 & 1,00 & 1,00 & 1,00 \\
\hline 15. Esquecimento do horário & 1,00 & 1,00 & 1,00 & 0,92 & 1,00 & 0,96 \\
\hline 16. Quando tem que comprar & 0,84 & 0,84 & 0,84 & 0,92 & 0,92 & 0,92 \\
\hline
\end{tabular}

Fonte: Dados da Pesquisa, 2019

Legenda: R/I= Redação e llustração; Cont.=Conteúdo; IVC= Índice de Validade de Conteúdo da Mensagem
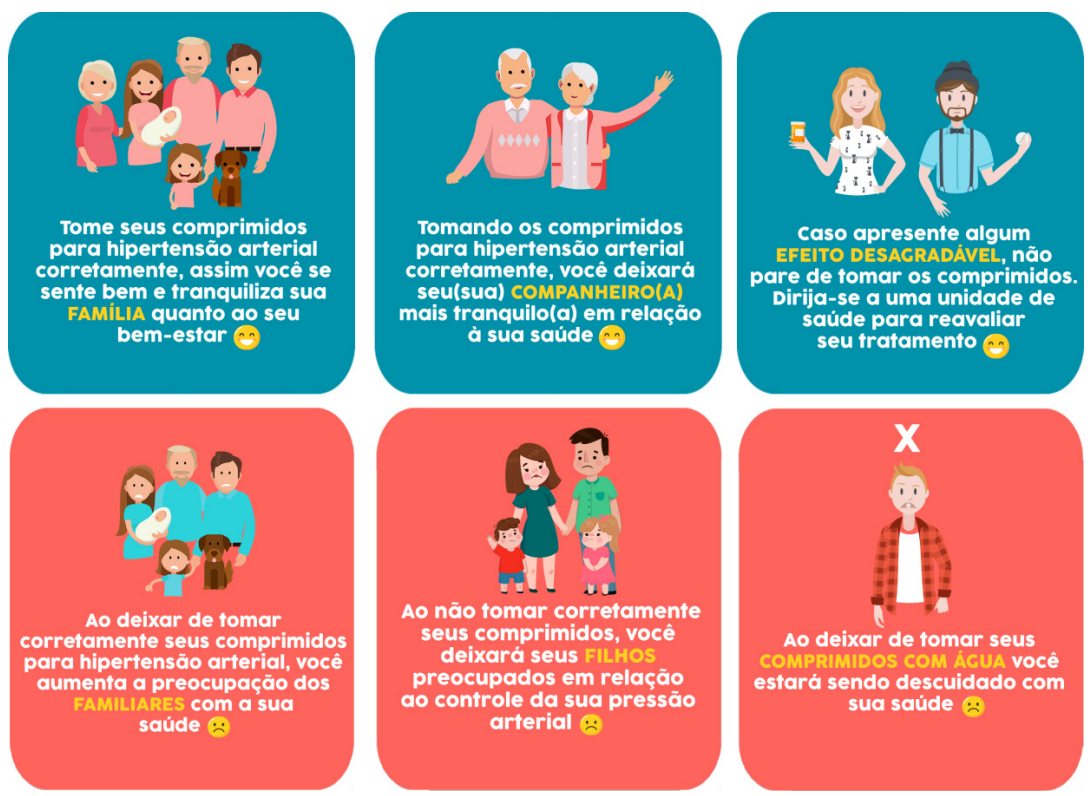

Figura 2. Imagens das mensagens persuasivas positivas e negativas após modificações realizadas com sugestões dos especialistas. Campina Grande/Paraíba, Brasil, 2019.

Fonte: Elaboração própria 
Importante ressaltar que, após a avaliação dos pesquisadores acerca das mensagens positivas e negativas que faziam menção às crenças de facilidade e/ou dificuldade de aderir ao tratamento pelo "Baixo custo" e/ou "Quando tem que comprar os comprimidos", foram excluídas a mensagem positiva número 16 e as negativas números 14 e 16. Tais conclusões ocorreram em virtude da inviabilidade de elaborar argumentos persuasivos adequados a uma população que apresenta dificuldades socioeconômicas para aquisição dos medicamentos, diversas vezes relatadas como um problema que vai muito além do seu controle volitivo. Essas mensagens abordavam as crenças relacionadas às dificuldades de aquisição dos fármacos, como, por exemplo, quando têm que comprar devido a estes não serem disponibilizados gratuitamente, mesmo que sejam com baixo custo, considerando o contexto de baixa renda dos entrevistados.

Consoante com tal ponderação, foram consideradas válidas, em relação ao seu conteúdo e aparência, 29 mensagens ilustradas (15 positivas e 14 negativas). Importa salientar que, em decorrência dos elevados índices de concordância entre os especialistas para a quase totalidade dos itens, houve apenas um ciclo de avaliação. Os recursos audiovisuais estão disponíveis, na íntegra, no canal do Laboratório de Tecnologias do Cuidar - TecSaúde, Youtube ${ }^{\circledR}$. A comunicação audiovisual pode ser acessada pelo link https://youtu.be/85aF_JcDqHU, e as mensagens persuasivas, no seguinte endereço: https:// youtu.be/dTGICgpC0I8.

\section{DISCUSSÃO}

De acordo com a TCP, a intenção é preditora imediata do comportamento, e intervenções delineadas a partir das crenças comportamentais, normativas ou de controle podem influenciar as intenções na direção desejada do comportamento. ${ }^{3-5}$ Assim, foram construídas a comunicação audiovisual e mensagens persuasivas positivas e negativas, baseadas nas crenças de pessoas com $\mathrm{HAS}^{18}$ sobre o tratamento medicamentoso, com o intuito de fortalecer o comportamento saudável de tomada dos anti-hipertensivos e modular crenças negativas em positivas em relação a esse comportamento.

Comunicação audiovisual e mensagens persuasivas produzidas de modo atrativo, utilizando os recursos de imagens e som com linguagem persuasiva, são consideradas ferramentas estimulantes com potencial de êxito para influenciar a adoção de comportamentos em saúde..$^{7,8,14}$

Intervenções apenas informativas para o gerenciamento da hipertensão têm eficácia limitada, particularmente, entre populações desfavorecidas. ${ }^{24}$ Diante de constatações como essa, a utilização de vídeos apenas com informações sobre a doença, assim como mensagens escritas de cunho informativo, não é suficiente. ${ }^{25}$ Recursos construídos com base em fatores emocionais, que valorizem os aspectos pessoais, experienciais e culturais, devem ser desenvolvidos para motivar comportamentos corriqueiros, como a tomada dos anti-hipertensivos, proposta elencada para este estudo.
Nesse processo, a validação de conteúdo do material elaborado pelos autores, por especialistas de diferentes áreas e, ainda, com a participação de profissionais experientes em cuidados à pessoa com HAS de diferentes regiões brasileiras, interessados em contribuir no aprimoramento das tecnologias construídas, foi essencial para o seu desenvolvimento, pois, apesar de o conteúdo ser estruturado intencionalmente por crenças locais, pontos abordados no material se fazem presentes em outras regiões do Brasil, como na região Sudeste, ${ }^{1}$ valorando a riqueza do produto.

Observou-se que os recursos audiovisuais elaborados obtiveram boas avaliações por parte dos especialistas em relação à clareza, pertinência e abrangência. Cabe salientar que cada sugestão foi analisada, mesmo que os aspectos avaliados tenham obtido IVC $\geq 0,80$ tanto nas mensagens como no vídeo e, quando cabíveis, as recomendações foram atendidas para favorecer a aceitação e compreensão pelo público-alvo. Os resultados corroboram outras investigações que empregaram a validação de conteúdo na construção de tecnologias de cuidado para promoção da saúde em doenças crônicas. ${ }^{26,27}$

Como demonstrado em estudo anterior, ${ }^{18}$ a escolha inicial do profissional médico para ilustrar a crença, tanto no vídeo como nas mensagens, ocorreu em virtude de apenas esse membro da equipe de saúde ter sido citado como referente positivo para tratamento medicamentoso. Porém, acatando e concordando com a sugestão da maioria dos especialistas, que eram enfermeiros e contribuíam maciçamente para o acompanhamento dessa clientela nos diferentes níveis de assistência à saúde, essa imagem foi modificada.

Entretanto, tal resultado nos alerta para o necessário protagonismo do enfermeiro quanto ao seu papel de educador e da sua representatividade para os usuários. Acena-nos que, no cenário pesquisado, os usuários podiam não ter, na pessoa do profissional enfermeiro, uma referência de importância que pudesse influenciá-los na direção da adesão terapêutica. Assim, é necessário repensar o cuidado e as ações desenvolvidas, de modo a fortalecer o vínculo, na perspectiva de o enfermeiro se tornar um referente social importante quando o assunto se refere à saúde.

Dessa forma, ao ampliar os referentes positivos para além dos médicos, os indivíduos que acessarem esses recursos poderão refletir sobre a presença e a importância de outros profissionais na equipe de saúde como apoio às decisões favoráveis ao tratamento.

Quanto às mensagens e suas correspondentes ilustrações, também, foram modificadas de acordo com os apontamentos realizados pelos especialistas e, como se tratavam de crenças semelhantes e de um banco de vetores de imagens, optou-se por manter as mesmas ilustrações, com o objetivo de padronizar o material construído.

Convém destacar a importância do uso de abordagens persuasivas diversificadas para influenciar atitudes, crenças e intenções na adoção e promoção de comportamentos benéficos em saúde. ${ }^{11}$ Optou-se por apresentar as mensagens com enquadramento 
positivo e negativo, a fim de modelar os componentes da intenção comportamental de uso anti-hipertensivos em investigações futuras. Salienta-se que as mensagens negativas não têm o intuito de punição, mas conscientizar acerca de complicações indesejáveis ante a não adesão à terapêutica medicamentosa prescrita, reforçando a importância de realizar o comportamento.

Corroborando essa assertiva, ensaio clínico realizada na Coréia avaliou o efeito de mensagens positivas e negativas na educação para o autocuidado em pacientes diabéticos. Os achados evidenciaram que as mensagens negativas produziram efeitos mais fortes nas atitudes, no controle comportamental percebido e nas intenções em relação ao autocuidado comparadas às mensagens positivas. ${ }^{9}$

Assim, considera-se que a comunicação audiovisual e as mensagens persuasivas, construídas com base em princípios da TCP e persuasão, ${ }^{6}$ configuram-se um produto qualificado, pois sua estruturação foi ancorada em referencial teórico e metodológico compatível com o objeto de estudo, e contou-se com a apreciação de um comitê de especialistas, criteriosamente, selecionado para avaliá-las. Portanto, acredita-se que os recursos audiovisuais produzidos contribuirão para o cuidado do enfermeiro e equipe multidisciplinar na promoção e educação em saúde para o controle da HAS.

No futuro, este material será inserido em aplicativo que está sendo desenvolvido para smartphone, ferramenta que será testada e passará ao domínio público, podendo ser acessada gratuitamente pelo público-alvo. Nova pesquisa com delineamento experimental será conduzida, objetivando avaliar o efeito das comunicações persuasivas com a intenção (motivação) de conscientizar pessoas com HAS acerca da necessidade de usar anti-hipertensivos.

O material, após testagem, poderá ser utilizado pela equipe multiprofissional de saúde, envolvida no cuidado e acompanhamento de pessoas com HAS, especialmente pela equipe de enfermagem, que cuida diretamente e diariamente dessa clientela nos diferentes cenários de atuação. Por se tratar de uma tecnologia leve-dura, seu conteúdo pode ser aplicado de diferentes formas, uma vez que se trata de tecnologia de fácil aplicação e com baixo custo na sua disseminação.

Como dificuldade, aponta-se o acesso aos especialistas em mídias digitais e na TCP, em decorrência do baixo retorno às cartas-convite e e-mails solicitando a apreciação do conteúdo, o que limitou o quantitativo de participantes na pesquisa, embora tenha satisfeito as recomendações metodológicas para os estudos de validação por especialistas. Além disso, a ausência de tecnologias deste tipo na literatura dificulta comparar resultados, sendo este estudo pioneiro na área de enfermagem e cuidado de pessoas com HAS.

Finalmente, acredita-se que estes recursos tecnológicos poderão contribuir para motivar e fortalecer o comportamento saudável de tomar os anti-hipertensivos orais, na medida em que foram construídos com ênfase no reforço positivo das crenças do público-alvo para execução do comportamento.

\section{CONCLUSÃO E IMPLICAÇÕES PARA PRÁTICA}

A comunicação audiovisual e as mensagens persuasivas, delineadas com base nas crenças de pessoas com HAS e com suporte na Teoria do Comportamento Planejado, apresentam-se adequadas, claras, pertinentes, abrangentes e com capacidade de persuasão para motivar o uso de anti-hipertensivos orais.

Espera-se que os recursos audiovisuais sejam implementados pelo enfermeiro e demais profissionais de saúde como intervenção de cuidado nas ações educativas para potencializar a terapêutica medicamentosa prescrita para o controle clínico da HAS. Como recursos originais dinâmicos, apresentam elementos (imagens e sons) para instrumentalizar o conhecimento sobre a doença, reforçar, esclarecer informações e motivar o uso consistente dos medicamentos prescritos.

Como proposta futura, os pesquisadores almejam ensaio clínico com a utilização dos recursos audiovisuais construídos, a fim de averiguar o efeito da comunicação audiovisual e mensagens persuasivas com enquadramentos positivo e negativo na modulação da intenção comportamental de adoção do uso de anti-hipertensivos orais.

\section{CONTIRUBIÇÕES DOS AUTORES}

Desenho do estudo. Taciana da Costa Farias Almeida. Coleta de dados. Taciana da Costa Farias Almeida. Simone Helena dos Santos Oliveira. Análise de dados e interpretação dos resultados. Taciana da Costa Farias Almeida. Mailson Marques de Sousa. Bernadete de Lourdes André Gouveia. Simone Helena dos Santos Oliveira. Redação e revisão crítica do manuscrito. Taciana da Costa Farias Almeida. Mailson Marques de Sousa. Bernadete de Lourdes André Gouveia. Simone Helena dos Santos Oliveira. Aprovação da versão final do artigo. Responsabilidade por todos os aspectos do conteúdo e a integridade do artigo publicado. Taciana da Costa Farias Almeida. Mailson Marques de Sousa. Bernadete de Lourdes André Gouveia. Simone Helena dos Santos Oliveira.

\section{EDITOR ASSOCIADO}

\author{
Candida Caniçali Primo
}

\section{REFERÊNCIAS}

1. Campos CL, Pierin AM, Pinho NA. Hypertension in patients admitted to clinical units at university hospital: post-discharge evaluation rated by telefone. Einstein (Sao Paulo). 2017;15(1):45-9. http://dx.doi.org/10.1590/ s1679-45082017ao3862. PMid:28444088.

2. Pagès-Puigdemont N, Tuneu L, Masip M, Valls P, Puig T, Mangues MA. Determinants of medication adherence among chronic patients from an urban area: a cross-sectional study. Eur J Public Health. 2019;29(3):41924. http://dx.doi.org/10.1093/eurpub/cky259. PMid:30521035.

3. Ajzen I.The theory of planned behavior. Organ Behav Hum Decis Process. 1991;50(2):179-211. http://dx.doi.org/10.1016/0749-5978(91)90020-T.

4. Ajzen I. The theory of planned behaviour: reactions and reflections. Psychol Health. 2011;26(9):1113-27. http://dx.doi.org/10.1080/0887 0446.2011.613995. PMid:21929476.

5. Fishbein M, Ajzen I. Predicting and changing behavior: the reasoned action approach. New York, NY: Routledge; 2015.518 p. 
6. Cialdini RB. As armas da persuasão. Rio de Janeiro: Sextante; 2012 $304 \mathrm{p}$.

7. Norman P, Cameron D, Epton T, Webb TL, Harris PR, Millings A et al. A randomized controlled trial of a brief online intervention to reduce alcoho consumption in new university students: combining self-affirmation, theory of planned behaviour messages, and implementation intentions. Br J Health Psychol. 2018;23(1):108-27. http://dx.doi.org/10.1111/ bjhp.12277. PMid:28941040.

8. Tessier D, Sarrazin P, Nicaise V, Dupont J-P. The effects of persuasive communication and planning on intentions to be more physically active and on physical activity behaviour among low-active adolescents Psychol Health. 2015;30(5):583-604. http://dx.doi.org/10.1080/0887 0446.2014.996564. PMid:25493545.

9. Park J, Kim SH, Kim JG. Effects of message framing and health literacy on intention to perform diabetes self-care: A randomized controlled trial. Diabetes Res Clin Pract. 2020;161:108043. http://dx.doi.org/10.1016/j. diabres.2020.108043. PMid:32006642.

10. Stuchi RAG, Carvalho EC. Persuasão como estratégia para modificar as crenças nos comportamentos de risco para a doença arterial coronariana. Rev Min Enferm. 2008;12(3):295-302.

11. Kreps GL, Villagran MM, Zhao X, McHorney CA, Ledford C, Weathers $M$ et al. Development and validation of motivational messages to improve prescription medication adherence for patients with chronic health problems. Patient Educ Couns. 2011;83(3):375-81. http://dx.doi. org/10.1016/j.pec.2011.04.029. PMid:21602010.

12. Almeida ND, Roazzi A. Álcool e direção em universitários, comunicação persuasiva e prevenção. Psicologia (Cons Fed Psicol). 2014;34(3):71532. http://dx.doi.org/10.1590/1982-3703000632013.

13. Cunha BGF, Dias MR. Comunicações persuasivas e doação regular de sangue: um estudo experimental. Cad Saude Publica. 2008;24(6):1407-18. http://dx.doi.org/10.1590/S0102-311X2008000600021. PMid:18545766

14. Mendez RD, Rodrigues RC, Spana TM, Cornélio ME, Gallani MC, Pérez-Nebra AR. Validation of persuasive messages for the promotion of physical activity among people with coronary heart disease. Rev Lat Am Enfermagem. 2012;20(6):1015-23. http://dx.doi.org/10.1590/ S0104-11692012000600002. PMid:23258713.

15. Chopra HK, Ram CVS. Recent guidelines for hypertension: a clarion call for blood pressure control in India. Circ Res. 2019;124(7):984-6. http://dx.doi.org/10.1161/CIRCRESAHA.119.314789. PMid:30920927.

16. Malachias M, Plavnik FL, Machado CA, Malta D, Scala LCN, Fuchs S. 7th Brazilian Guideline of Arterial Hypertension: Chapter 1 - Concept, Epidemiology and Primary Prevention. Arq Bras Cardiol. 2016 set;107(3, Suppl 3):1-6. http://dx.doi.org/10.5935/abc.20160151. PMid:27819380.
17. Carling CLL, Kristoffersen DT, Oxman AD, Flottorp S, Fretheim A, Schu"nemann $\mathrm{HJ}$ et al. The effect of how outcomes are framed on decisions about whether to take antihypertensive medication: a randomized trial.PLoS One. 2010;5(3):e9469. http://dx.doi.org/10.1371/ journal.pone.0009469. PMid:20209127.

18. Almeida TCF, Sousa MM, Pessoa MSA, Sousa LS, Gouveia BLA Oliveira SHS. Crenças de indivíduos com hipertensão arterial sistêmica relacionadas ao tratamento medicamentoso. Rev Rene. 2019 set 25;20:e41585. http://dx.doi.org/10.15253/2175-6783.20192041585.

19. Fleming SE, Reynolds J, Wallace B. Lights... camera... action! A guide for creating a DVD/Video. Nurse Educ. 2009;34(3):118-21. http://dx.doi. org/10.1097/NNE.0b013e3181a0270e. PMid:19412052.

20. Hoffart N, Doumit R, Nasser SC. Use of storyboards as an active learning strategy in pharmacy and nursing education. Curr Pharm Teach Learn. 2016;6(8):867-84. http://dx.doi.org/10.1016/j.cptl.2016.08.010.

21. Lei № 9.610, de 19 de fevereiro de 1998 (BR). Altera, atualiza e consolida a legislação sobre direitos autorais e dá outras providências. Diário Oficial da União [periódico na internet], Brasília (DF), 20 fev 1998 [citado 2019 out 10]. Disponível em: http://www.planalto.gov.br/ ccivil_03/leis/L9610.htm

22. Coluci MZO, Alexandre NMC, Milani D. Construção de instrumentos de medida na área da saúde. Cien Saude Colet. 2015 mar;20(3):925-36. http://dx.doi.org/10.1590/1413-81232015203.04332013. PMid:25760132.

23. Alexandre NMC, Coluci MZO. Validade de conteúdo nos processos de construção e adaptação de instrumentos de medida. Cien Saude Colet. 2011;16(7):3061-8. http://dx.doi.org/10.1590/S1413-81232011000800006. PMid:21808894.

24. Bokhour BG, Fix GM, Gordon HS, Long JA, DeLaughter K, Orner $\mathrm{MB}$ et al. Can stories influence African-American patients' intentions to change hypertension management behaviors? A randomized control trial. Patient Educ Couns. 2016;99(9):1482-8. http://dx.doi.org/10.1016/j. pec.2016.06.024. PMid:27387121.

25. Sugita H, Shinohara R, Yokomichi H, Suzuki K, Yamagata Z. Effect of text messages to improve health literacy on medication adherence in patients with type 2 diabetes mellitus: a randomized controlled pilot trial. Nagoya J. Med. Sci. 2017;79(3):313-21. PMid:28878436.

26. Santiago JCS, Moreira TMM. Validação do conteúdo do livreto sobre excesso de peso para adultos com hipertensão. Rev Bras Enferm. 2019 fev;72(1):95-101. http://dx.doi.org/10.1590/0034-7167-2018-0105. PMid:30916273.

27. Ribeiro SA, Moreira AD, Reis JS, Soares AN, Géa-Horta T. Elaboração e validação de um folheto sobre diabetes para agentes comunitários de saúde. Rev Bras Enferm. 2020;73(4):e20180899. http://dx.doi. org/10.1590/0034-7167-2018-0899. PMid:32578729. 